\title{
ALTERNATIVE PATHS TO EARTH-MOON TRANSFER
}

\author{
CRISTIANO FIORILO DE MELO AND OTHON CABO WINTER
}

Received 28 December 2004; Revised 27 July 2005; Accepted 23 August 2005

The planar, circular, restricted three-body problem predicts the existence of periodic orbits around the Lagrangian equilibrium point L1. Considering the Earth-lunar-probe system, some of these orbits pass very close to the surfaces of the Earth and the Moon. These characteristics make it possible for these orbits, in spite of their instability, to be used in transfer maneuvers between Earth and lunar parking orbits. The main goal of this paper is to explore this scenario, adopting a more complex and realistic dynamical system, the four-body problem Sun-Earth-Moon-probe. We defined and investigated a set of paths, derived from the orbits around L1, which are capable of achieving transfer between lowaltitude Earth (LEO) and lunar orbits, including high-inclination lunar orbits, at a low cost and with flight time between 13 and 15 days.

Copyright (c) 2006 C. F. de Melo and O. C. Winter. This is an open access article distributed under the Creative Commons Attribution License, which permits unrestricted use, distribution, and reproduction in any medium, provided the original work is properly cited.

\section{Introduction}

Ever since the beginning of space exploration, the Moon has been the target of countless missions, and everything leads to believe that this will continue to be so. This certainty is focused on the recent discoveries of signs that point to the possible existence of ice at the lunar poles made by the American probes Clementine in 1994 [21] and Lunar Prospector in 1998 [10]. The estimates indicate that there may be some 10 billion tons of ice at the lunar poles. Therefore, if the existence of this ice were confirmed, future lunar bases would have a water source capable of sustaining life. This ice could also serve as a source for rocket fuel, by separating the water into hydrogen and oxygen, thus making the Moon into a trampoline for future manned interplanetary missions.

In this context, a special type of path, capable of direct transfer between a low-altitude Earth orbit (LEO) and a low-altitude Moon orbit, with flight time between 13 and 15 days, could be of great interest for future lunar missions. This paper presents the results 
of our research on this type of path, and shows that these paths could be a good alternative for an Earth-Moon transfer, and also other kinds of transfer in Earth-Moon system. The origin of these paths is related to a family of generally unstable direct orbits around the Lagrangian equilibrium point L1, known as the Family G. These paths are predicted by the circular, planar, restricted three-body problem associated with the Earth-Moon system (Broucke [8]). This is why we initially considered the three-body Earth-Moonprobe problem to establish a set of initial conditions, close to the Earth, and final conditions, close to the Moon, in such a way that these conditions were related through an empirical mathematical expression. Next, we considered a more complex and realistic dynamical problem, the four-body problem Sun-Earth-Moon-probe, where we take into account the eccentricity of the Earth's orbit as well as the eccentricity and inclination of the Moon's orbit. An empirical expression related to the initial and final conditions of the paths continued to exist. On the other hand, the consideration of a more complex dynamical system opened up new lines of investigation with respect to the probe's inclination and speed on its final approach to the Moon.

This paper is structured as follows. In Section 2, to facilitate comparison, we have quickly described two of the main conventional methods of Earth-Moon transfer and the method of gravitational capture. In Section 3, we describe the two dynamical systems utilized in this study. In Section 4, we discuss the main properties of these orbits for the restricted three-body problem and the four-body problem. In Section 5, we present, through a curve and an empirical mathematical expression, the set of paths capable of making the maneuver. In Section 6, we present some examples of missions that could be made using these paths, and finally, in Section 7, we present our conclusions and the perspective for future researches.

\section{Conventional methods of Earth-Moon transfers}

The calculation of a transfer path between the Earth and the Moon can only be made through numerical integration of the equations of motion [1]. These equations must take into account the Earth's, Moon's, and Sun's gravitational fields, and the mutual interactions between these bodies. In addition, given the complexity of the Moon's movement, a lunar mission must be planned and executed on an hour-by-hour and day-by-day basis. However, we can consider some approximations, based on the two-body and restricted three-body problem, to reach an initial estimate of the impulse needed to transfer a probe from the vicinity of the Earth to the vicinity of the Moon.

Next, we will describe two conventional methods used for preliminary analysis of the $\Delta V$ 's necessary for an Earth-Moon transfer and the gravitational capture method. Our goal is not to describe them rigorously, but rather to create a basis for comparison with the transfers that could be done by a set of paths derived from the unstable orbits around the Lagrangian equilibrium point L1; a set that we will present in Section 5.

2.1. Minimal energy path. It is possible to establish a sort of minimal energy path for a transfer between the Earth and the Moon based on the two-body Earth-probe and Moonprobe problems. This maneuver is divided into two parts. In the first part, the Moon's gravitational field is neglected, and in the second part, the Earth's gravitational field is 


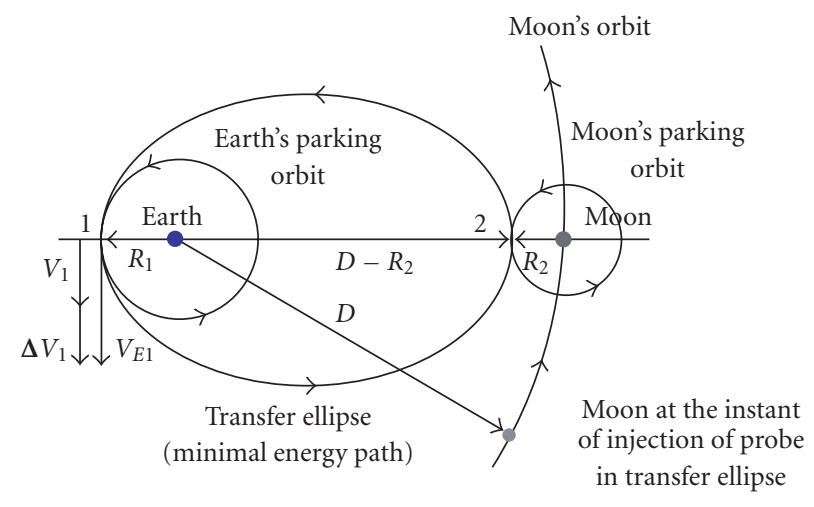

(a)

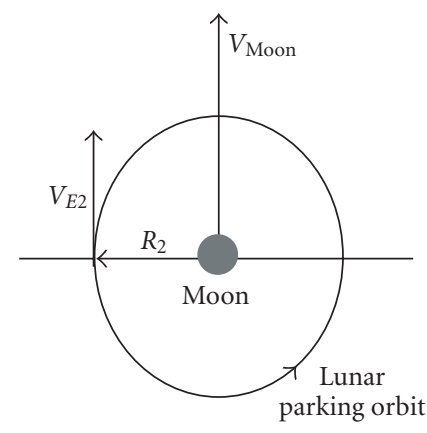

(b)

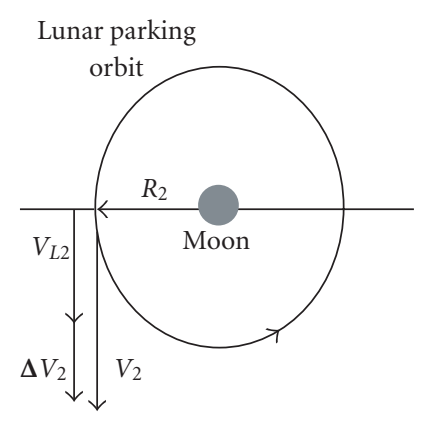

(c)

Figure 2.1. (a) Minimal energy transfer ellipse and the Earth's and Moon's circular parking orbits in geocentric system. (b) Velocities of the Moon and of the probe (in its apogee's transfer ellipse) in geocentric system. (c) Velocities of the probe relative to the Moon at the moment of application of the $\Delta V_{2}$ in selenocentric system. (Not to scale.)

neglected. This way, we can establish a simple procedure to estimate the impulses required for the maneuver based on Hohmann's transfer [1]. Figure 2.1 shows the minimal energy path, an ellipse, it is tangent to Earth's and Moon's parking orbits. The first part starts with the application of a $\Delta V_{1}$ when the vehicle attains the perigee's transfer ellipse. From Figure 2.1, we obtain the semimajor axis of transfer ellipse, $a_{\min }$, by

$$
a_{\min }=\frac{R_{1}+D-R_{2}}{2},
$$

where $R_{1}$ is the perigee's radius of the transfer ellipse and also of the Earth's parking orbit radius in the point of tangency with the transfer ellipse, $D=384400 \mathrm{~km}$ is the EarthMoon distance which we are considering constant, that is, we are considering the Moon to be in a circular orbit around the Earth, and $R_{2}$ is the Moon's parking orbit radius in the point of tangency between it and the transfer ellipse. The energy per unit of mass of 
4 Alternative paths to Earth-Moon transfer

this ellipse is

$$
\xi_{\min }=-\frac{G M_{\text {Earth }}}{2 a_{\min }}
$$

where $G=6.67 \times 10^{-11} \mathrm{~m}^{3} / \mathrm{s}^{2} \mathrm{~kg}$ and $M_{\text {Earth }}=5.9742 \times 10^{24} \mathrm{~kg}$ are the universal gravitational constant and the mass of the Earth, respectively. Thus, we can obtain the perigee's speed of the transfer ellipse, $V_{E 1}$, by

$$
V_{E 1}=\sqrt{2\left(\frac{G M_{\text {Earth }}}{R_{1}}+\xi_{\text {min }}\right)}
$$

and the apogee's speed of transfer ellipse, $V_{E 2}$, by

$$
V_{E 2}=\sqrt{2\left(\frac{G M_{\text {Earth }}}{D-R_{2}}+\xi_{\text {min }}\right)} .
$$

If we consider that the Earth's and Moon's parking orbits are circular with radius $R_{1}$ and $R_{2}$, respectively, the first impulse required to insert the vehicle in the transfer ellipse, $\Delta V_{1}$, is given by

$$
\Delta V_{1}=V_{E 1}-\sqrt{\frac{G M_{\text {Earth }}}{R_{1}}} .
$$

As we can see, the first part of the maneuver is equal to Hohmann's transfer. However, the second part of the maneuver corresponds to the application of a second impulse to insert the vehicle in lunar orbit and not to put it in a circular orbit with radius $D-R_{2}$. Therefore, the second part is different from Hohmann's transfer procedure, because the $\Delta V_{2}$ required for this maneuver should be applied in the opposite direction of the vehicle's motion. In order to calculate the magnitude of $\Delta V_{2}$, we should consider the apogee's vehicle speed, $V_{E 2}$, relative to the Moon. This speed we denote by $V_{L 2}$, and its magnitude is given by

$$
V_{L 2}=\left|V_{E 2}-V_{\text {Moon }}\right|
$$

where $V_{\text {Moon }}=1.023 \mathrm{~km} / \mathrm{s}$ is the Moon's orbital speed around the Earth (assuming circular orbit). The magnitude of $\Delta V_{2}$ is immediately calculated by

$$
\Delta V_{2}=\sqrt{\frac{G M_{\mathrm{Moon}}}{R_{2}}}-V_{L 2},
$$

where $M_{\text {Moon }}=7.3483 \times 10^{22} \mathrm{~kg}$ is the Moon's mass, and

$$
\Delta V_{\text {Total }}=\Delta V_{1}+\Delta V_{2}
$$




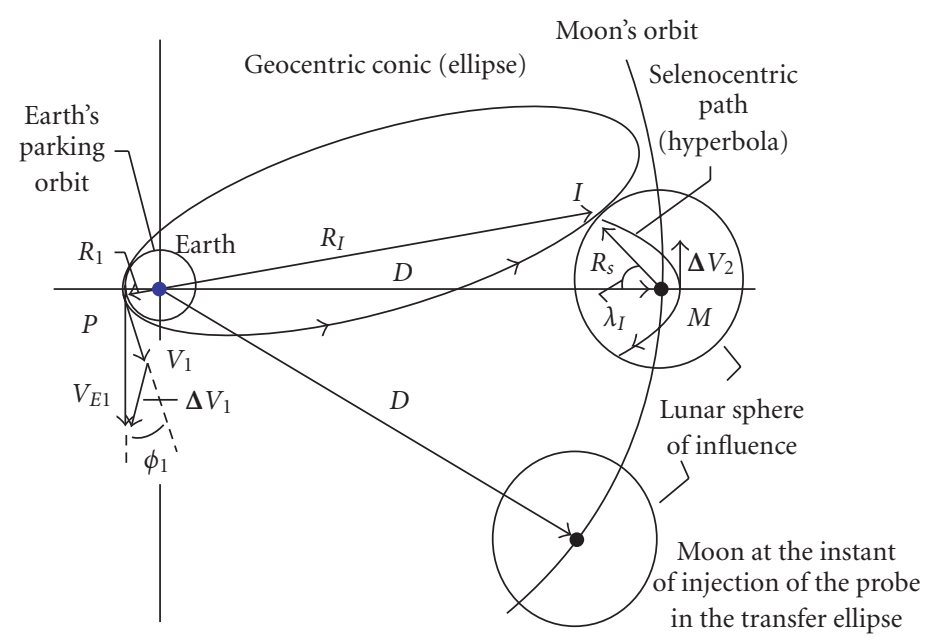

Figure 2.2. Geocentric conic of transfer (ellipse) and the transfer geometry seen in the geocentric coordinates system (not to scale).

The flight time for this maneuver is simply half of the orbital period of the transfer ellipse, that is,

$$
T_{\text {Flight }}=\pi \sqrt{\frac{a_{\text {min }}^{3}}{G M_{\text {Earth }}}} .
$$

The procedure described in this section is the simplest method to estimate the magnitude $\Delta V_{\text {Total }}$ for a direct maneuver transfer between the Earth and the Moon. But, it makes use of two-body dynamics, for this reason, the $\Delta V_{\text {Total }}$ given by (2.8) is not sufficient to take the vehicle to the final lunar orbit desired, requiring additional $\Delta V$ 's to complete the mission [18].

2.2. Patched-conic approach. Similar to the transfer via minimal energy ellipse, the patched-conic approach is also divided into two parts. The basic idea is to consider the probe as being under the influence of Earth's gravitational field only since the moment of launch in transfer path, called geocentric conic, until the instant it reaches the Moon's sphere of influence (first part). At this time, the probe is considered to be under the influence of the Moon's gravitational field only (second part). The transition from geocentric motion to selenocentric motion occurs naturally along a finite arch of the probe's path when it is in the region where the geocentric conic touches or intercepts the Moon's sphere of influence; whose radius is $R_{S}=66300 \mathrm{~km}$. Figure 2.2 illustrates the basic geometry of patched-conic transfer. In this figure, we can see the first part of the maneuver that is, leaving the Earth's parking orbit and the geocentric-conic tangency of the Moon's sphere of influence. Theoretically, the geocentric conic could be an ellipse, a parabola, or a hyperbola, but generally, ellipses require the smallest $\Delta V$ 's for the maneuver. Figure 2.2 
also shows the second part of the maneuver in which the probe gets inside the lunar sphere of influence.

The transfer starts when a $\Delta V_{1}$ is applied injecting the probe into geocentric conic, this occurs at point $P$ (Figure 2.2). At the point at which the probe reaches the Moon's sphere of influence, point $I$ (Figure 2.2), it begins to move under the influence of the Moon. When the probe reaches the periselenium of the selenocentric path, point $M$ (Figure 2.2), a $\Delta V_{2}$ must be applied to conclude the maneuver.

In order to calculate the elements of the geocentric and selenocentric paths and the $\Delta V$ 's required to transfer, we need to know at least four independent initial quantities, or three independent initial quantities and one intermediary (on the lunar sphere of influence), or final quantities. The search for these quantities is an iterative procedure. Particularly, a convenient set for these quantities is $R_{1}, V_{E 1}, \phi_{1}$, and $\lambda_{I}$ (see Figure 2.2), where $R_{1}$ is the radial distance at the point $\mathrm{P}, V_{E 1}$ is the injection speed into geocentric conic, $\phi_{1}$ is the flight-path angle, and $\lambda_{I}$ specifies the point at which the geocentric trajectory crosses the lunar sphere of influence.

Thus, if the values attributed to $R_{1}, V_{E 1}, \phi_{1}$, and $\lambda_{I}$ lead the probe to periselenium equal to the radius of the final lunar orbit planed, the procedure is complete, otherwise, a new search for other values for $R_{1}, V_{E 1}, \phi_{1}$, and $\lambda_{I}$ must be started until the selenocentric path reaches periselenium equal to the radius of the final lunar orbit desired.

The procedure described above is a good approximation for preliminary mission analysis, but, in practice, errors occur in the encounter with the Moon's sphere of influence due to the disturbance of the Sun's gravitational field, and also due to the Moon's gravitational field in the first part of the maneuver and the Earth's in the second part of the maneuver. The calculations needed to reach the values of the $\Delta V$ 's are extensive and for this reason, we will not demonstrate them here. For more information, please consult [1].

2.3. Methods of Earth-Moon transfer by gravitational capture. The phenomenon of gravitational capture can be understood as the mechanism by which an object, subject only to gravitational forces, approaches a celestial body at a low speed, relative to this body, in such a way that the object can be captured, and then temporarily orbits around that body. For the capture to be permanent, a dissipative force must act upon the object, such as atmospheric drag, for example. This phenomenon has been considered by various researchers to explain the origin of planetary satellites, see Murison [15] and Brunini [9].

Gravitational capture is possible for the general three-body problem. Usually, the initial distance between the three bodies is infinite, and after the approach, the distance between two of them remains limited. For the restricted three-body problem, the dynamical system considered in the great majority of the studies found in the literature, the third body (particle) approaches one of the primary bodies from an infinite or finite distance. After the approach, the distance between the particle and one of the primary bodies varies between well-determined values, and the primary-particle orbital energy remains negative as long as the capture lasts, as this is a temporary phenomenon.

Transfer methods based on the phenomenon of gravitational capture cannot be called conventional, especially due to the wealth of information that they offer from the 
semianalytic point of view. But the mechanism can be conveniently exploited to place spacecraft in orbit around celestial bodies as a technique to reduce fuel consumption. Some of the first studies of this theme were conducted by Belbruno [2-5], Belbruno and Miller [6, 7], Miller and Belbruno [14], Krish et al. [12]. Other more recent interesting studies in this area are those of Yamakawa [23], Koon et al. [11], Ocampo [17], Prado $[19,20]$, and Winter et al. [22]. They all study the mechanism by which spacecraft can be inserted into orbit around the Moon. There are also studies related to gravitational capture applied to low-consumption transfers to the moons of Jupiter, Saturn, and Uranus, for example, Lo and Ross [13].

The mechanism of gravitational capture was applied in 1991 in the Japanese Hiten mission [6].

\section{Dynamical systems}

3.1. Restricted three-body problem (R3BP). This problem, well known in the literature-see, for example, Murray and Dermott, [16]—considers three bodies, $m_{1}, m_{2}$, and $m_{3}$, with $m_{3}$ being a particle with negligible mass that does not influence the other two bodies, which have preponderant mass and are called primary. These, in turn, have circular, coplanar orbits around the center of mass that is common to both; they maintain a constant distance from each other, and also have the same angular velocity relative to this center of mass. Due to these characteristics, it is useful to study the R3BP adopting a system of references whose origin is fixed in the center of the mass common to the primary bodies, with axes $x$ and $y$ rotating with the same angular velocity as the first bodies. This system is called a barycentric rotating or synodic system, and in it, the bodies $m_{1}$ and $m_{2}$ remain fixed over the $x$-axis, while $m_{3}$ moves on the $x y$-plane. The system is normalized, considering its reduced mass, $\mu$, as unitary mass, that is, $\mu=\mu_{1}+\mu_{2}=G\left(m_{1}+m_{2}\right)=1$, where $G$ is the universal gravitational constant. The constant distance between the masses $m_{1}$ and $m_{2}$ is also considered equal to 1 . Thus, the coordinates of $m_{1}$ and $m_{2}$ are $\left(-\mu_{2}, 0\right)$ and $\left(\mu_{1}, 0\right)$, respectively. The equations of motion for the third body in the synodic system are

$$
\begin{gathered}
\ddot{x}=2 \dot{y}+x-\left[\mu_{1} \frac{\left(x-\mu_{2}\right)}{r_{13}^{3}}+\mu_{2} \frac{\left(x-\mu_{1}\right)}{r_{23}^{3}}\right], \\
\ddot{y}=2 \dot{x}+x-\left[\frac{\mu_{1}}{r_{13}^{3}}+\frac{\mu_{2}}{r_{23}^{3}}\right] y
\end{gathered}
$$

with

$$
\begin{aligned}
& r_{13}^{2}=\left(x+\mu_{2}\right)^{2}+y^{2}, \\
& r_{23}^{2}=\left(x-\mu_{1}\right)^{2}+y^{2},
\end{aligned}
$$

where, considering the Earth-Moon-probe system, $\mu_{1}=\mu_{\text {Earth }}=0.9878494$ and $\mu_{2}=$ $\mu_{\text {Moon }}=0.0121506$ are the mass parameters for the Earth and the Moon, respectively, $r_{13}$ is the Earth-particle distance and $r_{23}$ is the Moon-particle distance. Equations (3.1) 


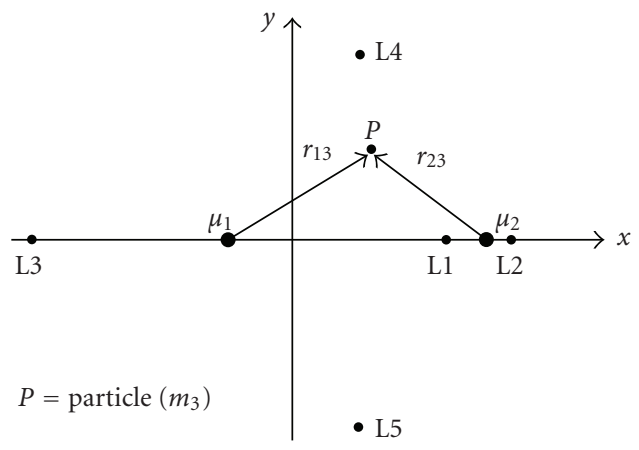

Figure 3.1. Synodic reference system and the relative location of the Lagrangian equilibrium points for Earth-Moon system.

do not have an analytical solution, but they do have symmetry properties that guarantee the existence of periodic orbits in the synodic system (Broucke [8]; and Murray and Dermott [16]), such as the Family G orbits that we investigated in this study.

The R3BP also has another interesting property which is the existence of five equilibrium points called Lagrangian equilibrium points, symbolized by the letter L. When a particle is placed on these points with a null velocity relative to the origin of the synodic system, it remains there indefinitely. Figure 3.1 illustrates this synodic system and the relative location of the five Lagrangian equilibrium points for Earth-Moon system.

3.2. Four-body problem. In our numerical simulations, we also considered as dynamical system the four-body problem in three-dimensional space. Thus, for a system of fixed Cartesian coordinates, in which the position of a certain body is given by the vector $\mathbf{x}_{k i}=$ $\left(x_{k 1}, x_{k 2}, x_{k 3}\right) \in \mathbb{R}^{3}$, the equations of motion are given by

$$
\ddot{x}_{k i}=\sum_{\substack{j=1 \\ j \neq k}}^{4} \frac{\mu_{j}}{r_{j k}^{3}}\left(x_{j i}-x_{k i}\right),
$$

where $k=1,2,3,4$;

$$
r_{j k}=\left|\mathbf{x}_{j}-\mathbf{x}_{k}\right|=\left[\sum_{i=1}^{3}\left(x_{j i}-x_{k i}\right)^{2}\right]^{1 / 2}
$$

is the distance between the $k$ th and the $j$ th bodies, $\mu_{j}$ is the mass parameter for the $j$ th body, and with $i=1,2,3$ denoting the three coordinates of the fixed Cartesian system. Equation (3.3) represents 12 second-order differential equations and expresses the fact that acceleration of a given body is the result of the sum of the forces exercised by the other three bodies. This means that (3.3) takes into account the mutual interactions between the four bodies in the system. Now, if we associate indexes 1 to the Sun, 2 to the Earth, 3 to the Moon, and 4 to the probe, and still consider that the system is normalized 
based on the Earth's and Moon's mass, so that $\mu_{2}+\mu_{3}=1$, then we will have $\mu_{1}=\mu_{\text {Sun }}=$ 328904.4747, $\mu_{2}=\mu_{\text {Earth }}=0.987849396$, and $\mu_{3}=\mu_{\text {Moon }}=0.012150604$. Considering that a lunar probe's mass can vary between some hundreds of kilograms and some tons, its mass parameter, $\mu_{4}$, will be at order of $10^{-23}$ or $10^{-24}$, and even with (3.3) taking into account the mutual interactions between the four bodies considered, the probe would not influence the motion of the other three. For this reason, the terms of (3.3), which contain $\mu_{4}$, were suppressed. This is the four-body Sun-Earth-Moon-probe problem, and the word restricted could precede it, without a loss of generality, given the order of the size of $\mu_{4}$. The aforementioned normalization is completed adopting the average distance between the Earth and the Moon, $384400 \mathrm{~km}$, as a unit of measurement.

The eccentricity of the Earth's orbit, $e_{2}$, the eccentricity, $e_{3}$, and the inclination, $i_{3}$, of the Moon's orbit were included in the system via initial conditions, thus bringing the system closer to reality. The values for these elements are $e_{2}=0.0167, e_{3}=0.0549$, and $i_{3}=5.1454^{\circ}$ (relative to the ecliptic).

\section{Properties of the Family G orbits}

Considering the R3BP, the Family G generally has short-range orbits around L1, longrange orbits that pass a few kilometers from the Earth's surface and a few dozen kilometers from the Moon's surface, and even orbits that present a loop. In the synodic system, the first two kinds of orbits have initial conditions of the following type:

$$
\left(x_{0}, 0,0, \dot{y}_{0}\right)
$$

while the third kind has initial conditions of the following type:

$$
\left(x_{0}, 0,0,-\dot{y}_{0}\right)
$$

Figure 4.1 exhibits an example of each kind, seen in the synodic system. We can observe that for the first two kinds of orbits, point $x_{0}$ is between the Earth and L1; and point $x_{1}$, which corresponds to the first passage by the particle along the $x$-axis of the coordinate system under consideration, is located between L1 and the Moon. For the third kind, $x_{0}$ is also between the Earth and L1, and $x_{1}$ is located to the left of the Earth, between the letter and L3. All three kinds of orbits are unstable.

The kinds of paths illustrated in Figure 4.1(b) are those paths capable of making a direct transfer between the Earth and the Moon. Figure 4.2 exhibits, for the restricted threebody problem, a path of this type, a quasiperiodic orbit. In Figure 4.2(a), it is seen in the synodic system, in Figure 4.2(b), in the geocentric system, together with the Moon's orbit, and in Figure 4.2(c), we have a zoom showing a loop given by the path of the Moon's orbit.

Figure 4.3 shows for the four-body problem the orbit obtained with the same initial conditions as in Figure 4.2. Note that with the final approach to the Moon, the probe leaves the Moon's orbital plane. This fact allows the probe to be inserted into a highly inclined lunar orbit.

Note that for the initial conditions (4.1) and (4.2), the particle is always on the $x$ axis, between the Earth and the Moon, at $t=0$. In this manner, considering a probe in 


\section{Alternative paths to Earth-Moon transfer}

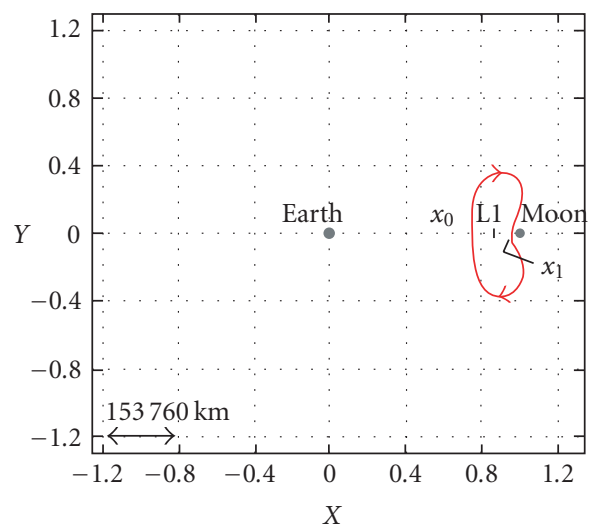

(a)

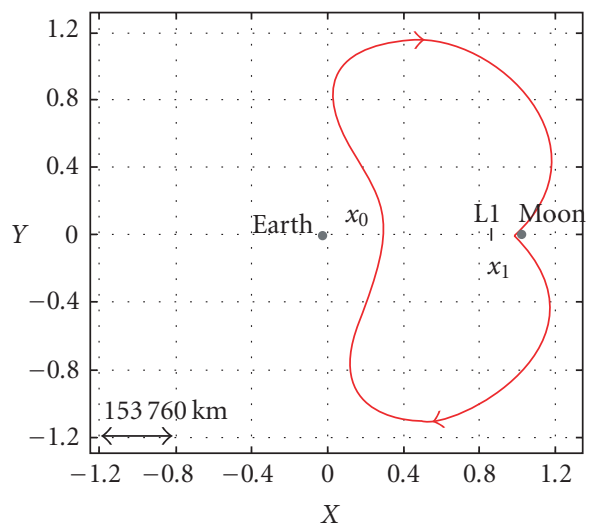

(b)

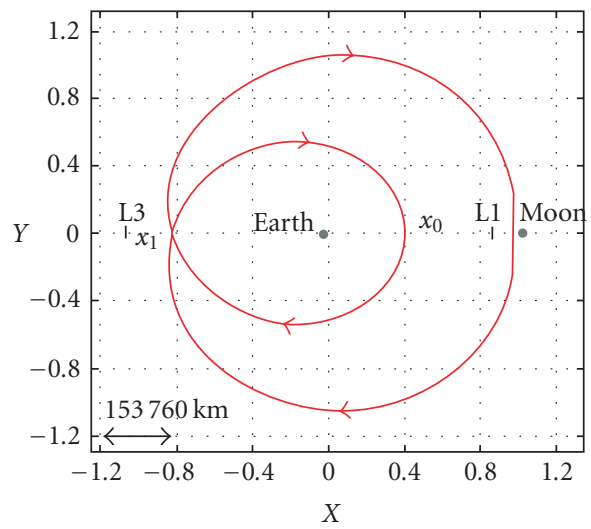

(c)

Figure 4.1. Family $\mathrm{G}$ of periodic orbits obtained considering the R3BP: (a) short range and (b) long range, with $x_{0}$ between the Earth and L1 and with $x_{1}$ between L1 and the Moon. (c) Orbit that has loops with $x_{0}$ between the Earth and L1, and with $x_{1}$ between the Earth and L3. 


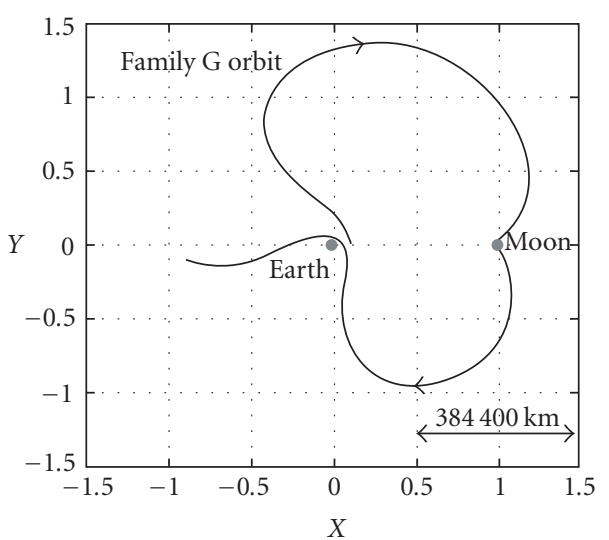

(a)

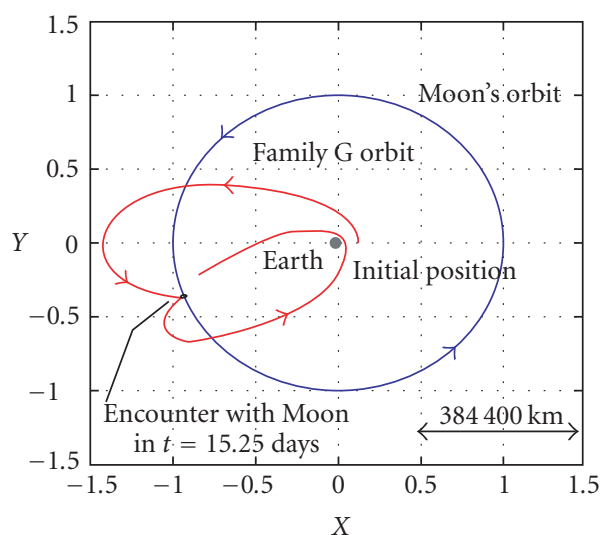

(b)

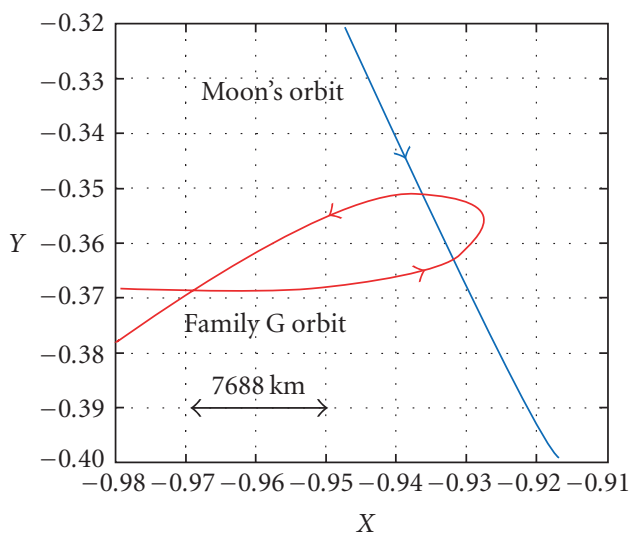

(c)

Figure 4.2. Unstable path of the Family G and Moon's orbit (R3BP). (a) Earth-Moon rotating synodic system, (b) geocentric system, and (c) zoom showing the paths of the probe and the Moon in the encounter region. The initial condition in geocentric system is $\left(x_{0} ; 0 ; 0 ; \dot{y}_{0}\right)=$ $(42370 \mathrm{~km} ; 0 ; 0 ; 4.169 \mathrm{~km} / \mathrm{s})$. 


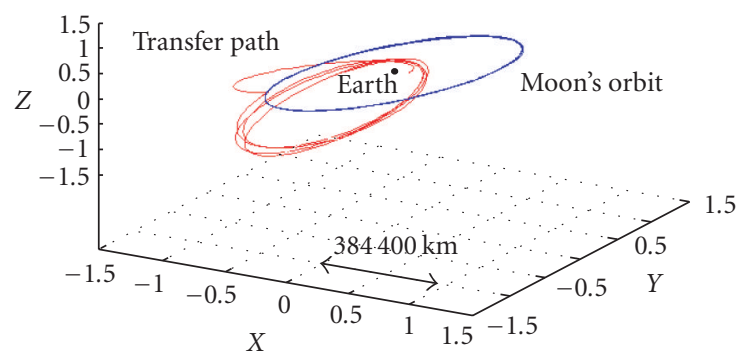

Figure 4.3. Path of transfer spatial view in the geocentric system obtained considering the four-body problem Sun-Earth-Moon-probe. The initial condition in the geocentric system is $\left(x_{0} ; 0 ; 0 ; \dot{y}_{0}\right)=$ $(42370 \mathrm{~km} ; 0 ; 0 ; 4.169 \mathrm{~km} / \mathrm{s})$.

a terrestrial parking orbit, the injection impulse to acquire a transfer path could only be applied when the Earth, probe, and Moon were all lined up, in this order. For example, for a terrestrial parking orbit with an altitude equal to $200 \mathrm{~km}$, there would be a launching window open every 1.47 hours, a time frame that is not restrictive to using these orbits for transfer maneuvers between the Earth and the Moon.

\section{Definition of the set of transfer paths}

In order to select the paths capable of achieving transfer between a terrestrial and lunar parking orbits, we consider that the probe departs always from a circular orbit around the Earth. We consider altitudes for this orbit, $H_{T}$, varying between 160 and $20000 \mathrm{~km}$. Then, we select only those paths that reach the periselenium with altitudes, $H_{L}$, between 0 and $100 \mathrm{~km}$. This procedure allows us to find a curve that demonstrates the relation between the injection speed for acquisition of a transfer path, $V_{I}$, and the altitude of the terrestrial circular parking orbit, $H_{T}$, for transfer path's periselenium altitude, $H_{L}$, less than $100 \mathrm{~km}$ from Moon's surface, including collision paths. But if we consider $160 \leq H_{T} \leq 700 \mathrm{~km}$, an empirical mathematical expression that relates $V_{I}$ and $H_{T}$ for $H_{L} \leq 100 \mathrm{~km}$ can be written. Considering the four-body problem Sun-Earth-Moon-probe, the mathematical expression is given by

$$
V_{I}=-8.5 \times 10^{-4} H_{T}+11.10100+\delta
$$

The interval $160 \leq H_{T} \leq 700 \mathrm{~km}$ was chosen taking into account the capacity of present launch vehicles. The set of transfer paths can be seen in Figure 5.1(a), and the set defined by (5.1) in Figure 5.1(b), which is a zoom of Figure 5.1(a) for $160 \leq H_{T} \leq 700 \mathrm{~km}$. Note that for this interval, we have a black band defining the region and not a line, this fact justifies the last term, $\delta$, in (5.1). Figure 5.2 shows a zoom of the diagram of Figure 5.1(b) for $240 \leq H_{T} \leq 245 \mathrm{~km}$. In this figure it is possible to verify the extreme sensitivity of periselenium altitude with injection speed in achieving the transfer path. This structure exists in all the intervals studied, and can be expressed taking into account small variations in $\delta$. These variations are presented in Table 5.1.

The numerical results found for PR3C are quite similar to those of the four-body problem, in terms of the curve as well as the mathematical expression. That is, graphs $V_{I}$ versus 


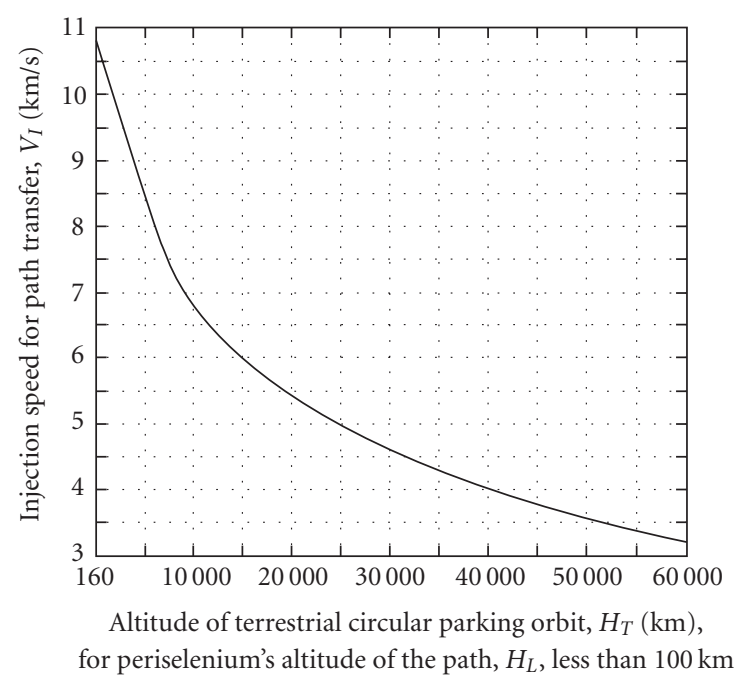

(a)

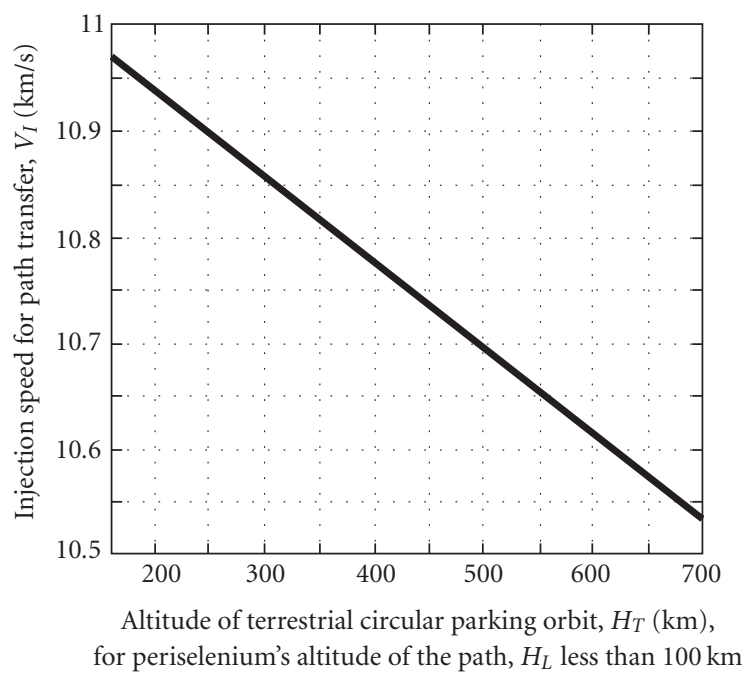

(b)

Figure 5.1. Injection speed, $V_{I}$, versus the altitude of the Earth's parking orbit, $H_{T}$, for paths with periselenium, $H_{L}$, less than $100 \mathrm{~km}$. (a) $160 \leq H_{T} \leq 20000 \mathrm{~km}$ and (b) $160 \leq H_{T} \leq 700 \mathrm{~km}$.

$H_{T}$ for the PR3C are practically identical to those shown in Figures 5.1(a) and 5.1(b), and the mathematical expression $V_{I}=V_{I}\left(H_{T}\right)$ is almost the same as the one in (5.1). For this reason, the results were not shown here. In fact, the differences in the values for $V_{I}$ between the two dynamical systems only begin with the third decimal position in $\mathrm{km} / \mathrm{s}$. Nonetheless, there is a significant difference between the defined paths for each dynamical 


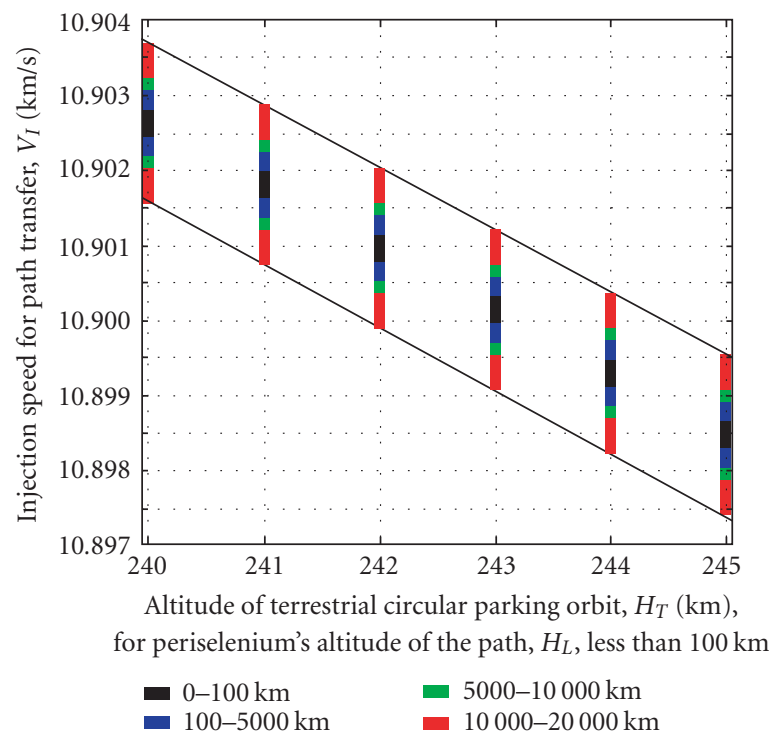

Figure 5.2. Injection speed, $V_{I}$, versus the altitude of the Earth's parking orbit, $H_{T}$, for paths with various altitudes of periselenium, $H_{L}$, indicated using a color code.

Table 5.1. Relations between $\delta$ and periselenium altitude.

\begin{tabular}{lc}
\hline Interval of variation of $\delta(\mathrm{km} / \mathrm{s})$ & Altitude of periselenium $(\mathrm{km})$ \\
\hline$|\delta| \leq 1.46 \times 10^{-4}$ & $0-100$ \\
\hline $1.47 \times 10^{-4} \leq \delta \leq 4.86 \times 10^{-4}$ & $100-5000$ \\
$-4.86 \times 10^{-4} \leq \delta \leq-1.47 \times 10^{-4}$ & $5000-10000$ \\
\hline $4.86 \times 10^{-4}<\delta \leq 7.28 \times 10^{-4}$ & \\
$-7.28 \times 10^{-4} \leq \delta<-4.86 \times 10^{-4}$ & $10000-20000$ \\
\hline $7.28 \times 10^{-4}<\delta \leq 1.10 \times 10^{-3}$ & \\
$-1.10 \times 10^{-3} \leq \delta<-7.28 \times 10^{-3}$ & \\
\hline
\end{tabular}

system. This difference is in the position of the periselenium of the transfer path; for the PR3C, it is obviously located in the Moon's orbital plane. However, for the four-body problem, the paths defined graphically by Figure 5.1, and also by (5.1), leave the Moon's orbital plane in their final approximation-see path shown in Figure 4.3, for example. In this manner, the osculating lunar orbit that contains the periselenium of the transfer path has inclination relative to the Moon's orbital plane and longitude of the ascending node that is different from zero. The inclination of this orbit varies between 40 and 42 degrees for transfer paths that leave terrestrial parking orbits with $160 \leq H_{T} \leq 5000 \mathrm{~km}$; for $5000<H_{T} \leq 20000 \mathrm{~km}$, the inclination declines gently until it reaches 29 degrees. These inclination values do not take into account the fact that the Moon's equator has an inclination of 6.5 degrees relative to its orbital plane. The longitude of the osculating orbit's ascending node also varies little, between 115 and 117 degrees, for those paths that 


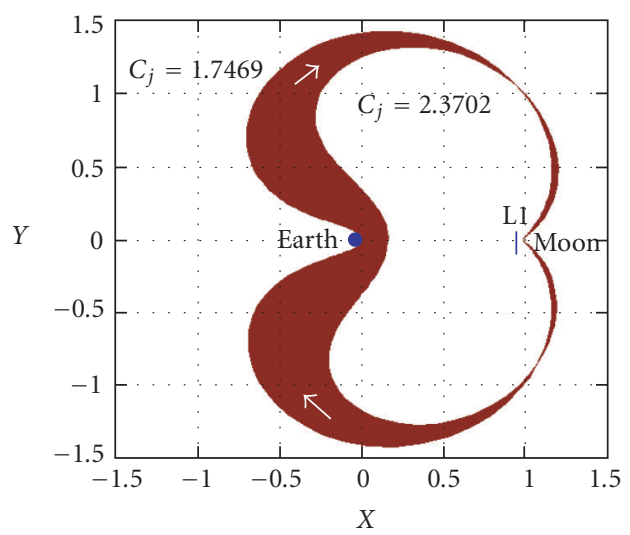

(a)

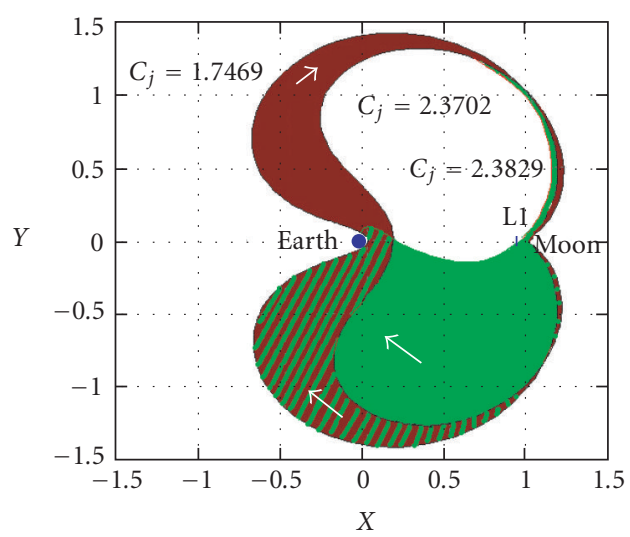

(b)

Figure 5.3. "Linking areas" between the Earth and the Moon in the synodic system for the PR3C with (a) $160 \leq H_{T} \leq 20000 \mathrm{~km}$ and $H_{L} \leq 100 \mathrm{~km}$ and (b) $160 \leq H_{T} \leq 20000 \mathrm{~km}$ and $100<H_{L} \leq$ $20000 \mathrm{~km}$.

leave terrestrial parking orbits with $160 \leq H_{T} \leq 5000 \mathrm{~km}$, and decreases gently until 108 degrees for paths with $5000 \leq H_{T} \leq 20000 \mathrm{~km}$.

It is interesting to observe that, in spite of the instability of the paths observed for the two dynamical systems under consideration, they define what we can call "links" between the Earth and the Moon. In order to better understand this, we will consider two paths obtained for PR3C: the first having $H_{T}=160 \mathrm{~km}, V_{I}=10.969 \mathrm{~km} / \mathrm{s}$, that is, in the geocentric coordinates system, $\left(x_{0} ; 0 ; 0 ; \dot{y}_{0}\right)=(6610 \mathrm{~km} ; 0 ; 0 ; 10.969 \mathrm{~km} / \mathrm{s})$, the altitude of the periselenium $H_{L}=10 \mathrm{~km}$ and $C_{J}$ (Jacob's constant) $=1.7469$; and the second having $H_{T}=20000 \mathrm{~km}, V_{I}=3.261 \mathrm{~km} / \mathrm{s},\left(x_{0} ; 0 ; 0 ; \dot{y}_{0}\right)=(20000 \mathrm{~km} ; 0 ; 0 ; 3.261 \mathrm{~km} / \mathrm{s})$, $H_{L}=96 \mathrm{~km}$, and $C_{J}=2.3702$. Note that these paths correspond exactly to the extremes of the interval considered for $H_{T}$. The "linking areas" in the synodic system, one on the departure and one on the return, defined by these two paths, are illustrated in Figure 5.3(a). 


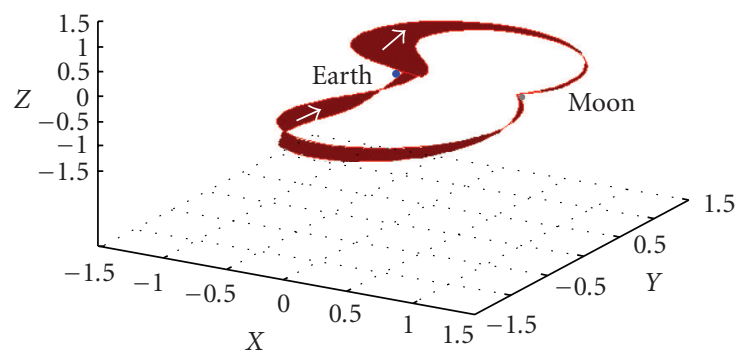

(a)

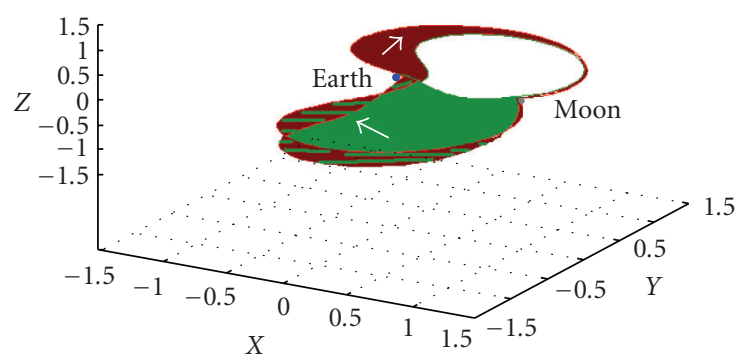

(b)

Figure 5.4. "Linking areas and canals" between the Earth and the Moon in the synodic system, obtained for the four-body problem with (a) $160 \leq H_{T} \leq 20000 \mathrm{~km}$ and $H_{L} \leq 100 \mathrm{~km}$ and (b) 160 $\leq H_{T} \leq 60000 \mathrm{~km}$ and $100<H_{L} \leq 20000 \mathrm{~km}$.

If we also consider that those paths which possess $100 \mathrm{~km}<H_{L} \leq 20000 \mathrm{~km}$ as the internal limit of the return "linking area" can be amplified, as shown in Figure 5.3(b), this guarantees faster Moon-Earth transfers.

By analogy, considering paths obtained for the four-body problem with the same initial conditions, that are, $H_{T}=160 \mathrm{~km}, V_{I}=10.967 \mathrm{~km} / \mathrm{s}, H_{L}=10 \mathrm{~km}, i$ (inclination of the osculating lunar orbit that contains the periselenium) $=41.44$ degrees, and $\Omega$ (longitude of the ascending node of the osculating lunar orbit) $=116.87$ degrees, and $H_{T}=$ $20000 \mathrm{~km}, V_{I}=3.259 \mathrm{~km} / \mathrm{s}, H_{L}=98 \mathrm{~km}, i=41.89$ degrees, and $\Omega=116.96$ degrees, the "area" of the departure journey continues to exist in the Moon's orbital plane. Nonetheless, the exit of the transfer path from the Moon's orbital plane in its final approximation introduces a "break in symmetry," with relation to that observed for the PR3C. The "area" of the return journey is transformed into a three-dimensional "canal" outside the Moon's orbital plane and its external limit is different from the external area of the departure journey, as shown in Figures 5.4(a) and 5.4(b).

\section{Flexibility of missions}

The properties of the set of paths found for the four-body Sun-Earth-Moon-probe problem can be conveniently exploited in at least three types of missions. The first type of mission corresponds to a direct transfer between a terrestrial parking orbit, with altitude 
varying between 160 and $20000 \mathrm{~km}$, and a lunar parking orbit, with altitude varying between 20 and $20000 \mathrm{~km}$, and inclination varying between 29 and 40 degrees, with application of only two impulses. The second type of mission utilizes the instability inherent to the paths and gain in inclination to insert them into lunar parking orbits with other inclinations, including polar orbits, at a low cost, applying a $\Delta V_{\text {director }}$ at mid-journey to direct the path in such a way that the lunar osculating orbit which contains the periselenium has inclinations greater than 40 degrees, or lower than 29 degrees, depending on the mission, of course. Finally, the third type of mission takes advantage of the instability of the paths and the "break in symmetry" caused by their exit from the Moon's orbital plane during the final approximation. The idea to be exploited is to launch a probe or satellite, towards the Moon in a path defined by (5.1), from a low-altitude terrestrial orbit (LEO), with $H_{T} \leq 700 \mathrm{~km}$, for example, and to take advantage of the gain in inclination and the return linkage "canal" to place the satellite in high-altitude terrestrial orbits and high inclinations, including polar orbits, possibly reducing the cost of this maneuver. Such a maneuver also requires the application of a $\Delta V_{\text {director }}$ during mid-flight to adequately direct the path.

Following we will discuss an example of the first type of mission, that is, a direct transfer between a low-altitude terrestrial parking orbit (LEO) with $H_{T}=320 \mathrm{~km}, V_{I}=10.834$ $\mathrm{km} / \mathrm{s}$, and a lunar parking orbit with $H_{L}=84.7 \mathrm{~km}, i=41.15$ degrees, and $\Omega=116.84$ degrees. The $\Delta V_{1}$ required for the first impulse, the injection impulse that will place the probe into the transfer path, is the difference between $V_{I}$ and the velocity of the terrestrial parking orbit, if we consider the parking orbit as circular, we have

$$
\Delta V_{1}=V_{I}-\sqrt{\frac{G M_{\text {Earth }}}{R_{1}}}=3.108 \mathrm{~km} / \mathrm{s} \text {, }
$$

where $R_{1}$ is the Earth's parking orbit radius in the point of tangency with transfer path $R_{1}=R_{T}+H_{T}$, with $R_{T}$ being the Earth's average radius $(6370 \mathrm{~km})$. The $\Delta V_{2}$ required for the second impulse, the insertion impulse into the lunar orbit, will correspond to the difference between the velocity of the probe in the periselenium of the transfer path, $V_{P}$, and the velocity of the planed lunar orbit at the point of its application. Supposing that the plane around the Moon is circular, we have

$$
\Delta V_{2}=V_{P}-\sqrt{\frac{G M_{\text {Moon }}}{R_{P}}}=0.920 \mathrm{~km} / \mathrm{s} .
$$

The value for $V_{P}$ is found through numerical integration, for this example $V_{P}=2.560$ $\mathrm{km} / \mathrm{s}, R_{P}=R_{L}+H_{L}$, with $R_{L}$ being the average radius of the Moon $(1738 \mathrm{~km})$. In the periselenium of the transfer path, the probe's velocity is always perpendicular to the Moon-probe position vector, which facilitates the insertion into a circular lunar orbit. The $\Delta V_{\text {Total }}$ is

$$
\Delta V_{\text {Total }}=\Delta V_{1}+\Delta V_{2}=4.028 \mathrm{~km} / \mathrm{s}
$$


The flight time is 13.92 days. The cost of the maneuver calculated by Hohmann is $\Delta V_{\text {Total }}=3.910 \mathrm{~km} / \mathrm{s}$ and by patched conic is $\Delta V_{\text {Total }}=4.118 \mathrm{~km} / \mathrm{s}$, and the flight time is of the order of 5 days for both. As we can see, the $\Delta V_{\text {Total }}$ via the transfer path as defined by (5.1) is greater than the $\Delta V_{\text {Total }}$ for the same maneuver via Hohmann (around $3 \%$ ) and less than the $\Delta V_{\text {Total }}$ via patched conic (around 2.2\%). However, as we saw in Section 2, these methods are based strictly upon the dynamics of the two-body Earthprobe and Moon-probe. Numerical simulations considering the PR3C or the four-body problem show that the $\Delta V_{\text {Total }}$ via Hohmann and patched conic are not sufficient to conclude the maneuver, requiring corrective $\Delta V$ 's to compensate for the disturbance caused by the Sun's and Moon's gravitational fields (first part of the maneuver) and of the Sun and the Earth (second part). These corrective $\Delta V$ 's can increase the cost of the maneuver by $5 \%$. On the other hand, the $\Delta V_{\text {Total }}$ via the transfer path defined by (5.1) already takes these disturbances into account.

\section{Conclusions}

In this study, we have been able to verify the existence of a well-defined set of paths, derived from the direct periodic orbits around the Lagrangian equilibrium point L1 (Family G [8]), and capable of carrying out, among other things, a direct transfer maneuver between low-altitude terrestrial and lunar parking orbits. This set was graphically defined starting with injection velocity $V_{I}$, or velocity required to acquire the transfer path, versus the altitude of the terrestrial parking orbit, $H_{T}$, for $160 \leq H_{T} \leq 20000 \mathrm{~km}$ and with $H_{L}$ (altitude of the lunar parking orbit) $\leq 100 \mathrm{~km}$ (Figures 5.1 and 5.2), and via an empirical mathematical expression for $160 \leq H_{T} \leq 700 \mathrm{~km}$ (5.1). The dynamical systems considered were the PR3C and the four-body Sun-Earth-Moon-probe problems, to which the eccentricity of the Earth's orbit and the eccentricity and inclination of the Moon's orbit were taken into account.

In addition to the direct transfer between the Earth and the Moon, the paths defined by the graphs in Figures 5.1 and 5.2 can also be used to insert a probe into lunar orbits with high inclinations, including polar orbits, thanks to the instability and to the fact that they leave the Moon's orbital plane in their final approximation. It is also possible to exploit this gain of inclination to transfer, at a low cost, a probe or a satellite from a lowaltitude terrestrial orbit (LEO), to an orbit with a much higher altitude, with or without high inclinations. This maneuver is possible because the set of investigated paths defines "linking areas" and/or "canals" between the Earth and the Moon, as shown in Figures 4.3, 5.3 , and 5.4 .

With respect to the cost of the direct transfer maneuver, when compared to the conventional methods of Hohmann and patched conic, we see that the paths studied have $\Delta V_{\text {Total }}$ that are very close to those obtained by these methods. However, the $\Delta V$ 's found via Hohmann and patched conic are not sufficient to conclude the maneuver, because they do not take into account the disturbances of the gravitational fields of the Sun, Earth, and Moon during the entire maneuver. This makes it necessary to apply corrective $\Delta V$ 's to conclude the transfer, which can increase the cost of the maneuver by $5 \%$. Nevertheless, the disturbances of the Sun's, Earth's, and Moon's gravitational fields are present in the investigations that led to the set of paths herein defined. With respect to the flight time, 
the conventional methods are unbeatable, but a flight time between 13 and 15 may be acceptable in logistical missions, transfer of automatic probes, and even in some manned missions given the presumed savings.

When compared to the traditional gravitational capture transfer methods, in general, the cost of a maneuver carried out by a path defined in (5.1), for example, is greater (around 5\%). However, the flight times for gravitational capture transfers are very long, and are measured in months (7-16), while flight times for the paths herein presented do not exceed two weeks.

Therefore, we can conclude that this study reveals a set of alternative paths for transfer missions in the Earth-Moon system that, if properly exploited, could reduce the costs of some important maneuvers in the present context and also in the future of space exploration.

\section{Acknowledgment}

The authors are grateful to the FAPESP (Fundação de Amparo à Pesquisa do Estado de São Paulo) and the CNPq (Conselho Nacional para Desenvolvimento Científico e Tecnológico), Brazil.

\section{References}

[1] R. R. Bate, D. D. Mueller, and J. E. White, Fundamentals of Astrodynamics, Dover, New York, 1971.

[2] E. A. Belbruno, Lunar capture orbits, a method of constructing earth moon trajectories and the lunar gas mission, Proceedings of 19th AIAA/DGLR/JSASS International Electric Propulsion Conference, Colorado Springs, Colorado, 1987, AIAA-87-1054.

[3] __ Examples of the nonlinear dynamics of ballistic capture an escape in earth moon system, Proceedings of AIAA Astrodynamics Conference (Oregon, 1990), American Institute of Aeronautics and Astronautics, Washington, DC, 1990, pp. 179-184, AIAA-90-2896.

[4] __ Ballistic lunar capture transfer using the fuzzy boundary and solar perturbations: a survey, Proceedings of International Congress of SETI Sail and Astrodynamics (Turin, 1992), 1992.

[5] _ Through the fuzzy boundary: a new route the moon, The Planetary Report 7 (1992), no. 3, 8-10.

[6] E. A. Belbruno and J. K. Miller, A ballistic lunar capture trajectory for Japanese spacecraft Hiten, International Document JPL IOM 312/90.4-1731, Jet Propulsion Laboratory, California Institute of Technology, California, 1990.

[7] _ Ballistic lunar capture for the lunar observe, Internal Document IOM 212/90.4-1752, Jet propulsion Laboratory, California Institute of Technology, California, 1990.

[8] R. A. Broucke, Periodic orbits in the restricted three-body problem with earth-moon masses, Tech. Rep. 32-1168, Jet Propulsion Laboratory, California Institute of Technology, California, 1968.

[9] A. Brunini, On the satellite capture problem, capture and stability regions for planetary satellites, Celestial Mechanics \& Dynamical Astronomy. 64 (1996), no. 1-2, 79-92.

[10] W. C. Feldman, B. L. Barraclough, S. Maurice, R. C. Elphic, D. J. Lawrence, D. R. Thomsen, and A. B. Binder, Major compositional units of the moon: lunar prospector thermal and fast neutrons, Science 281 (1998), no. 5382, 1489-1493.

[11] W. S. Koon, M. W. Lo, J. E. Marsden, and S. D. Ross, Low energy transfer to the moon, Celestial Mechanics \& Dynamical Astronomy. 81 (2001), no. 1-2, 63-73.

[12] V. Krish, E. A. Belbruno, and W. M. Hollister, An investigation into critical aspects of a new form of low energy lunar transfer, the Belbruno-Miller trajectories, AIAA/AAS Astrodynamics Conference 
(South Carolina, 1990), American Institute of Aeronautics and Astronautics, Washington, DC, 1992, pp. 435-444, AIAA-1992-4581.

[13] M. W. Lo and S. D. Ross, Low energy interplanetary transfers using invariant manifolds of L1, L2 and halo orbits, Proceedings of AAS/AIAA Space Flight Mechanics Meeting (California, 1998), 1998.

[14] J. K. Miller and E. A. Belbruno, A method for construction of a lunar transfer trajectories using ballistic capture, Proceedings of 1st AAS/AIAA Annual Spaceflight Mechanics Meeting (Texas, 1991), 1991, pp. 97-109, AAS-91-1000.

[15] M. A. Murison, The fractal dynamics of satellite capture in the circular restricted three-body problem, Astronom. J. 98 (1989), no. 6, 2346-2359.

[16] C. D. Murray and S. F. Dermott, Solar System Dynamics, Cambridge University Press, Cambridge, 1999.

[17] C. A. Ocampo, Transfers to earth centered orbits via lunar gravity assist, Acta Astronaut. 52 (2003), no. 2, 173-179.

[18] A. F. B. A. Prado, Minimun fuel trajectories for the lunar polar orbiter, Revista Brasileira de Automação e Controle (SBA). 12 (2001), no. 2, 163-170 (portuguese).

[19] _ A numerical study of the gravitational capture in the bi-circular restricted four-body problem, Proceedings of 54th International Astronautical Congress (Bremen, 2003), 2003, IAC-03A.1.01.

[20] A. F. B. A. Prado and E. Vieira Neto, Orbital maneuvers using gravitational capture, nonlinear dynamics, chaos, control and their applications to engineering sciences, New Trends in Dynamics and Control (J. M. Baltazar, P. B. Gonçalves, and R. M. F. L. R. F. Brazil, eds.), vol. 3, 2002, pp. $109-128$.

[21] P. A. Regeon, P. R. Lynn, M. Johnson, and R. J. Chapman, The clementine lunar orbiter, Proceedings of 20th GIFU ISTS \& 11th IAS, Paper no. 96-e-40, 2002.

[22] O. C. Winter, E. Vieira Neto, and A. F. B. A. Prado, Orbital maneuvers using gravitational capture times, Advances in Space Research 31 (2003), no. 8, 2005-2010.

[23] H. Yamakawa, On earth-moon transfer trajectory with gravitational capture, Ph.D. dissertation, University of Tokyo, Tokyo, 1992.

Cristiano Fiorilo de Melo: Programa de Pós-graduação em Engenharia e Tecnologia Espaciais, Instituto Nacional de Pesquisas Espaciais (INPE), 12245-970 São José dos Campos, SP, Brazil

E-mail address: cristianofiorilo@terra.com.br

Othon Cabo Winter: Grupo de Dinâmica Orbital \& Planetologia, Universidade Estadual Paulista (UNESP), Campus Guaratinguetá, 12516-410 Guaratinguetá, SP, Brazil;

Programa de Pós-graduação em Engenharia e Tecnologia Espaciais, Instituto Nacional de Pesquisas Espaciais (INPE), 12245-970 São José dos Campos, SP, Brazil

E-mail address: ocwinter@feg.unesp.br 


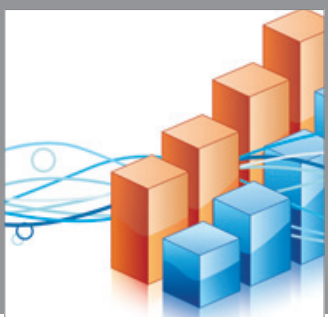

Advances in

Operations Research

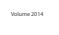

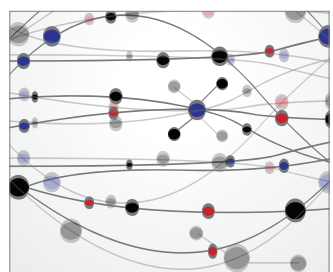

\section{The Scientific} World Journal
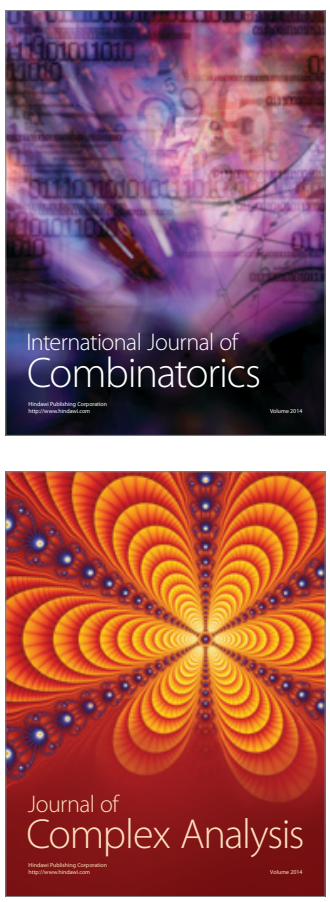

International Journal of

Mathematics and

Mathematical

Sciences
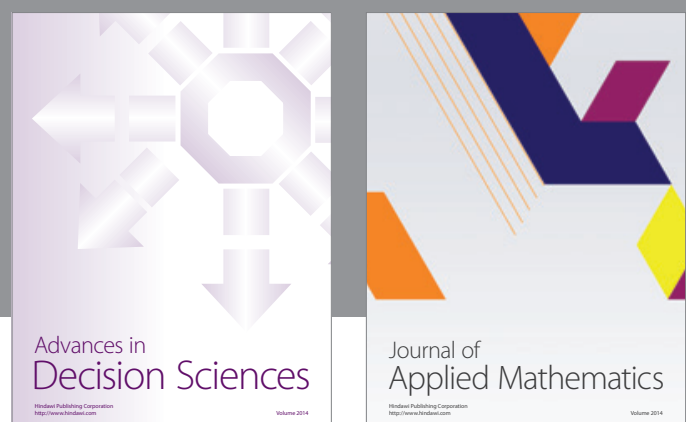

Journal of

Applied Mathematics
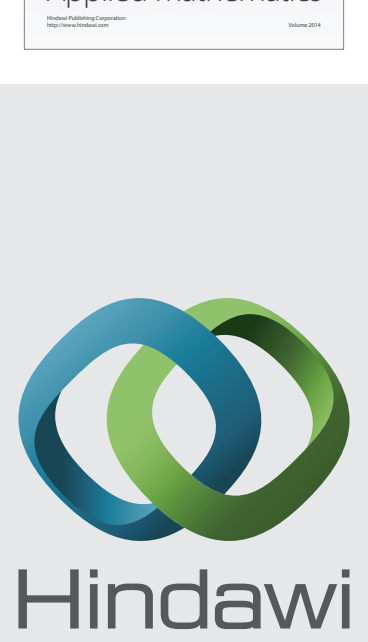

Submit your manuscripts at http://www.hindawi.com
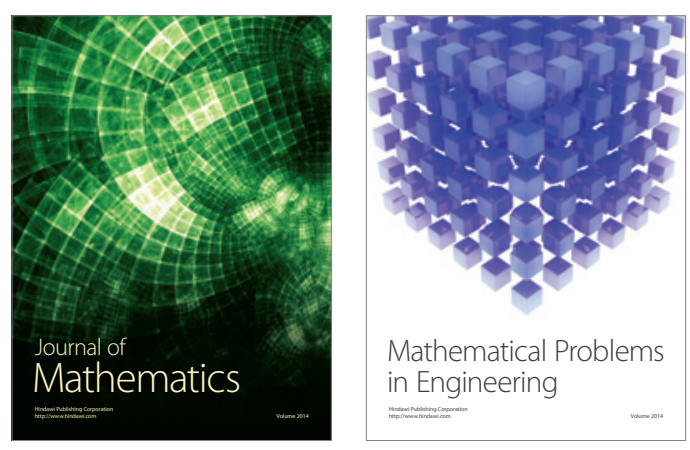

Mathematical Problems in Engineering
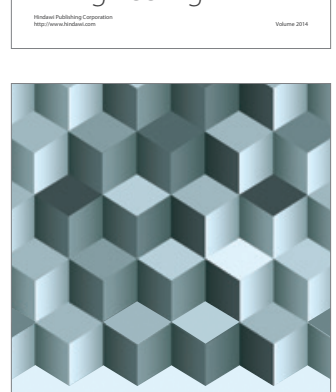

Journal of

Function Spaces
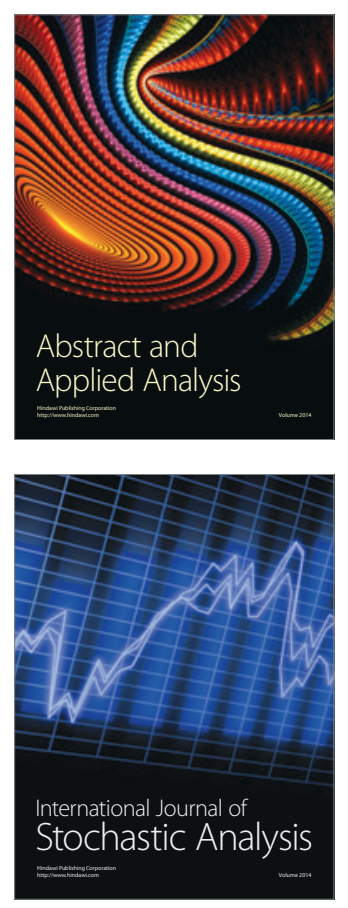

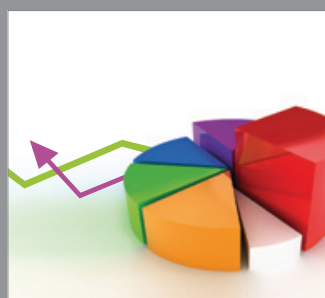

ournal of

Probability and Statistics

Promensencen
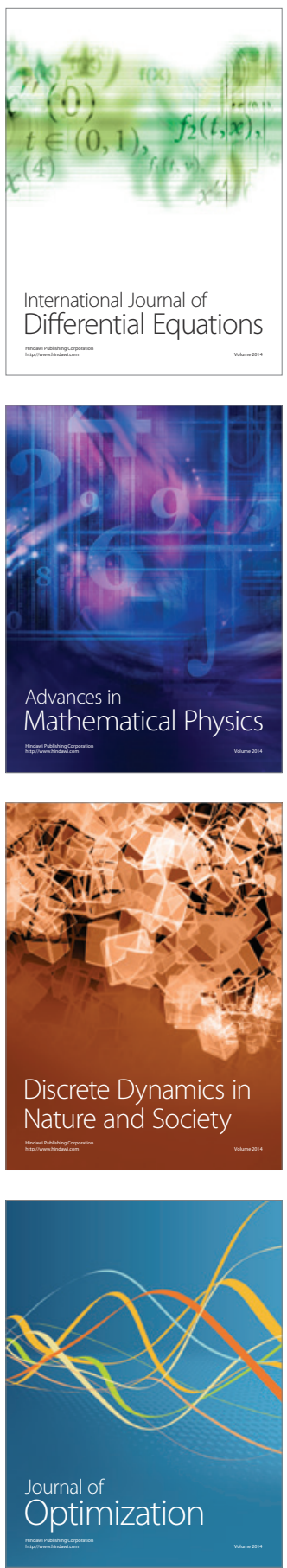